\title{
Technical Specification for Effective Next Generation Network Interconnection in Indonesia
}

\author{
Maman Abdurohman ${ }^{\mathrm{a}}$, Bambang Setia Nugroho ${ }^{\mathrm{b}}$ \\ ${ }^{a}$ School of Computing, Telkom University, Jl. Telekomunikasi No. 1, Bandung, 40257, Indonesia \\ E-mail: abdurohman@telkomuniversity.ac.id \\ ${ }^{b}$ School of Electrical Engineering, Telkom University, Jl. Telekomunikasi No. 1, Bandung, 40257, Indonesia \\ E-mail: bambangsetianugroho@telkomuniversity.ac.id
}

\begin{abstract}
This paper proposes technical specifications for effectively implementing the interconnection of Next Generation Network (NGN) in Indonesia. NGN is one of the current telecommunication infrastructure network technology trends. It provides a simpler concept with only two layers of service and transport. The NGN IP-based transport system can connect with various types of networks, which leads to low management costs by offering different kinds of services. Meanwhile, there are currently various types of telecommunication networks depending on the services provided, such as Public Switched Telephone Network (PSTN), IPv4 Internet as well as Public Switched Data Network (PSDN). PSTN is a circuit-switched voice communications network, and PSDN is a network for data-based communications with International Telecommunication Union (ITU)-T X.121 standards. Another type is a packet-switched based network that uses IPv4 addressing systems. Each network has its customers. One of the problems that arise, however, is how to transform the current network system to the NGN network effectively. The effectiveness of network transformation in the service provision for users is determined by the technical aspects used. Some of the technical aspects issues on implementation of NGN networks in Indonesia are the use of a protocol for signaling, coding standards, Quality of Service (QoS), numbering and addressing, and security. This paper proposes technical specifications for the effectiveness of NGN network implementation in Indonesia. Through the technical specification model, we propose, the risks that will arise in the implementation of NGN networks in Indonesia can be managed. Appropriate technical specifications have an essential role in the effectiveness of NGN network implementation in Indonesia.
\end{abstract}

Keywords-NGN; ITU-T; PSTN; PSDN; technical specification.

\section{INTRODUCTION}

The current trend of network technology, NGN, is a significant shift toward all IP-based networks. The new system based on packet-switched connection provides a much simpler system, including numbering system [1]. The Internet protocol as a data routing protocol is the key to convergence with a homogeneous and flat interconnection process. In the NGN network, only two layers are known, namely service and transport layers. Meanwhile, the diversity of access networks and physical layers are all handled by IP-based transport layers.

With such a simple system, the cost of the network management process is lower. In addition to this simplicity in management, a diversity of service layers is provided, including voice, text, video, and multimedia. In the implementation of the NGN network, is not only the diversity of services provided but also new opportunities, namely the existence of a new ecosystem of object-to-object communication or machine-to-machine communication (M2M). The new services provided by the NGN network create a new ecosystem that can be explored by telecom operators, the Internet of Things (IoT) and derivative technologies. This system can connect several sensors, actuators, and devices without human intervention. This trend is in the direction of automation and controlling system without human involvement. Such systems require low cost and management.

Implementation of the NGN network in a country is not as simple as its concept. The experiences of several countries in Europe in implementing a full-IP-based NGN network show that it is not yet entirely uniform [2]. Legacy networks, for instance, are still a constraint that cannot be eliminated immediately due to the still existing customers of the legacy system despite its not very significant number. One important aspect of an effective transformation from the existing network to the NGN network is an appropriate technical specification that suits the conditions of a country. 
This means that the selection of various technologies used in the NGN network to be implemented is tailored to the country's telecommunication environment, including Indonesia. It is, therefore, necessary to perform evaluations and studies to determine the technical specification model that is suitable for Indonesia.

The contribution of this paper is a proposal on technical specifications for the implementation of NGN networks in Indonesia. It is the result of evaluations performed on various technologies previously defined and applied. The determination of this specification was also based on the successful experiences of NGN implementation in other countries whose conditions are relatively like those of Indonesia, with some adjustments conducted. With the right technical specifications for the environment in Indonesia, this can minimize the risk of changes and improve the effectiveness of NGN network implementation in Indonesia.

\section{MATERIALS AND METHOD}

Other related parties have conducted several studies that has done a case study in the European Union for evaluating voice service based on IP-interconnection [2]. The paper explains the key issues related to IP interconnection: Point of Interconnection (PoI), Signaling protocol, codec, QoS, and redundancy. In this paper, the trend of PoI number has shown decreases with IP-based, and the dominant signaling protocol used is SIP, which supports all codec with various QoS parameters for various services as well as interconnections through direct network or hub network. In general, the survey has concluded that the perspectives of various stakeholders are almost identical. However, the characteristics in detail vary depending on the national situations.

In terms of the technical aspect, some papers have discussed in detail NGN architecture, covering its principle, function, and implementation [3]. This article introduces the IP Multimedia Subsystem (IMS) to improve features that are compatible with fixed and mobile network requirements. Also, in [4] the operation and management of NGN to interwork with existing networks, such as PSTN, satellites, broadcasting, etc., are discussed. Another paper [5] describes NGN supports multiple services and dynamic requirements that enable convergence between fixed and mobile networks. In [6], there is a detailed discussion about the problems encountered when designing, implementing, and validating the EuQoS system (heterogeneous network end-to-end QoS service) and recommend for improving user Quality of Experience (QoE). It is also stated that defining a transparent and useful end-user interface is very important in NGN. The framework describing the necessary aspects, and the relationships among these aspects are well described in [7].

\section{A. IP Interconnection}

Based on the definitions of GSMA [8] and BEREC [2], IP Interconnection can be understood as an interconnection model of traffic exchange using IP networks (voice, SMS, and data) from any mobile, fixed, or other providers using an IP based network. Interconnections on the IP network can be divided into 2 parts, i.e., interconnection on managed network and interconnection on the unmanaged network, as shown in Fig. 1.

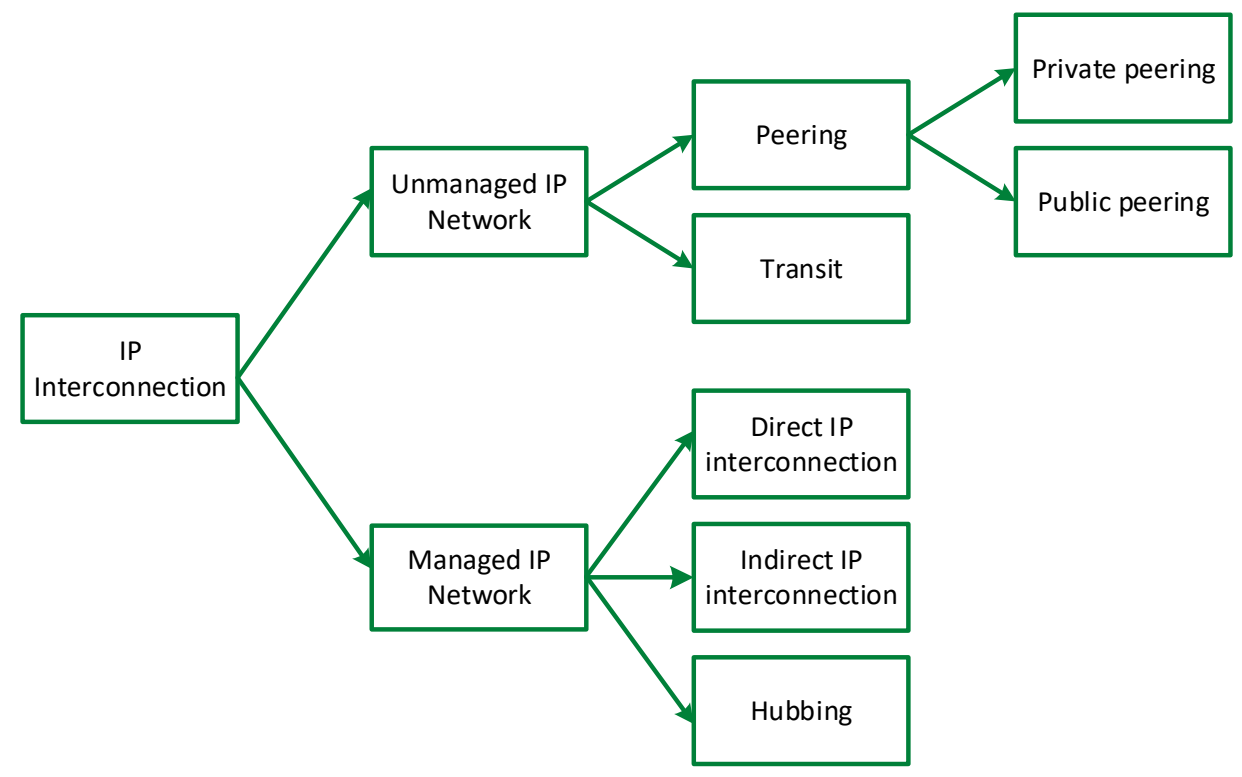

Fig. 1 Managed Network

\section{B. Interconnection on Unmanaged Network}

Interconnection on Unmanaged Network or also referred to as Best Effort is an interconnection that occurs in service without any traffic control and service quality. An example of this service is OTT (Over the Top); OTT utilizes the public internet access service provided by a network provider for its services to be used. Fig. 2. shows the managed service and unmanaged service on the IP network.

The difference between managed service/VoIP (green) and unmanaged service/OTT (yellow) can be seen in Fig. 2. It shows that the OTT service is only connected to the public internet network, while VoIP uses a dedicated network. The 
OTT service offers flexibility and portability, while VoIP offers quality assurance and security.

Interconnection on unmanaged networks, such as on the public internet, can be done directly (peering) or indirectly (transit). Fig. 3. shows the Internet interconnection as an example of the unmanaged network.

Peering is an Interconnection and bilateral agreement between two separate ISPs to exchange each other's traffic to their respective customers. Internet peering has special characteristics, such as that both entities have the same level of the tier where the technical and business arrangements are made bilaterally. Also, there are neither settlement process nor service level agreement. Both entities agree to receive traffic from each other's customers. Fig. 4. shows Peering interconnection.

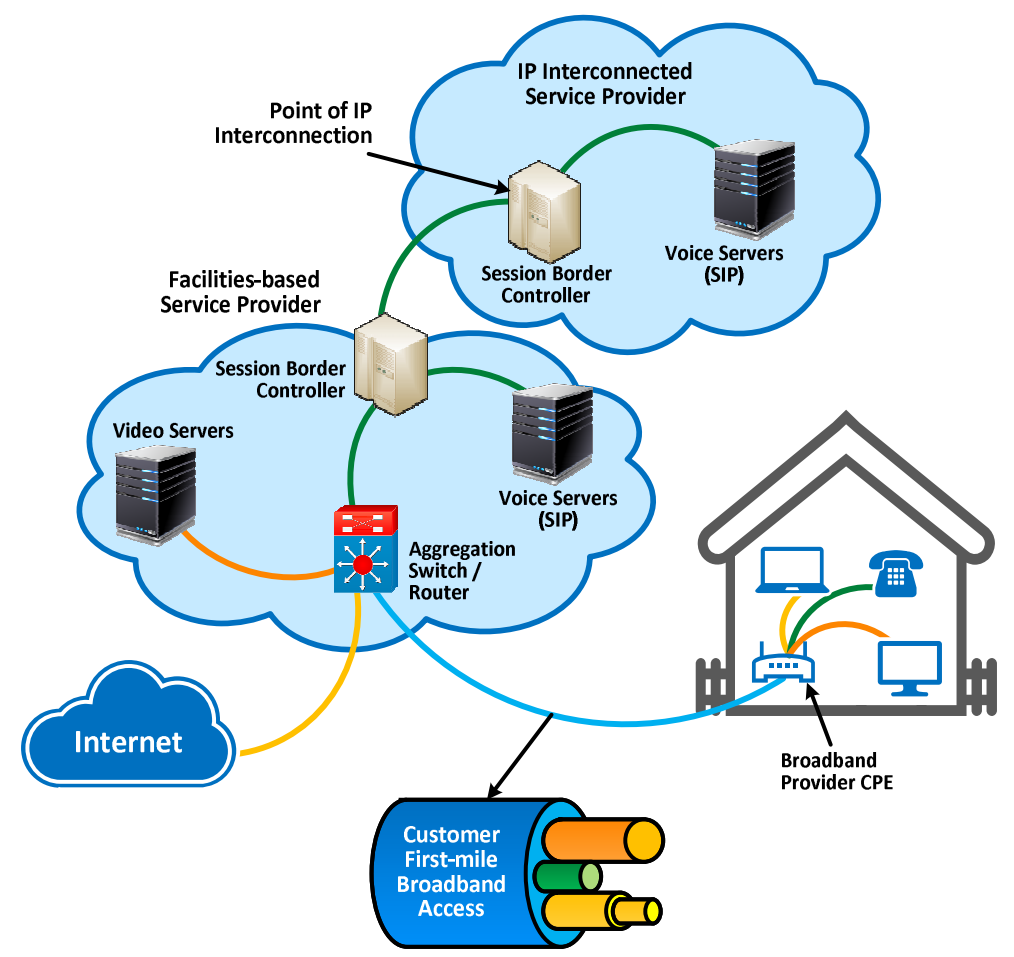

Fig. 2 Illustration of managed services and unmanaged services on an IP network [9]

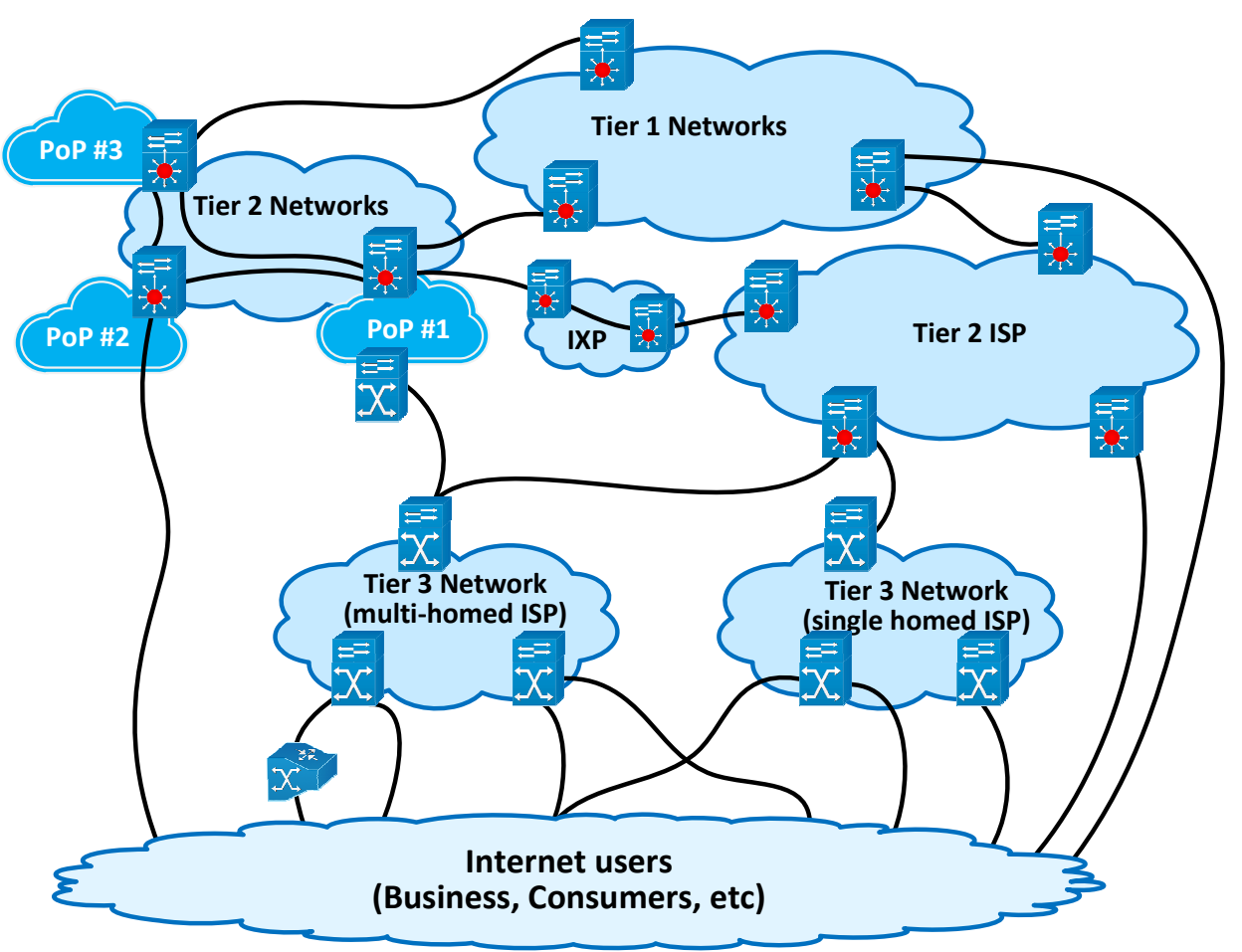

Fig. 3 Internet interconnection as an example of the unmanaged network 
Peering

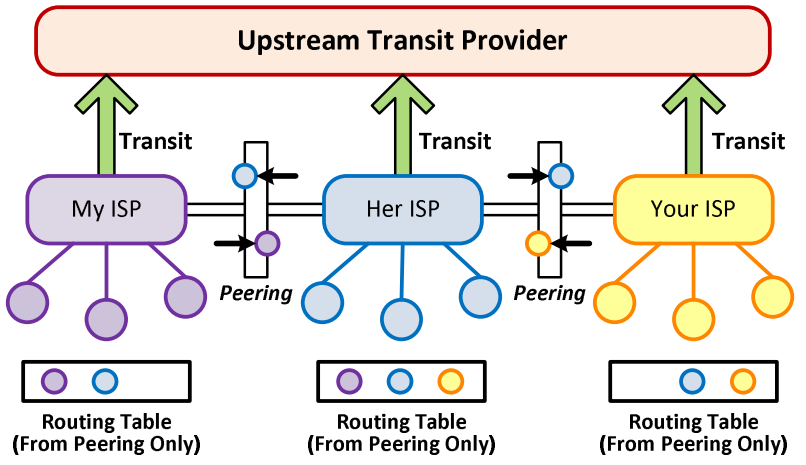

Fig. 4 Peering interconnection [10]

There are two types of peering systems such as private and public peering. Private peering, as shown in Fig. 5, involves connections between two networks through dedicated links using a gateway router. It can be said that private peering is an interconnection that occurs when no other party is not identified, which may affect the VoIP service between two networks.

The interconnections that occur when VoIP traffic is combined with another IP traffic that comes from the Internet allowing the gateway to be reached by other unidentified networks that may affect the quality of the service. Public peering involves more than two operators that connect to a public peering point. Usually, the government provides the interconnection points. This condition is suitable when there are many operators because building private peering is difficult. Fig. 6. shows public peering schemes consisting of five different operators.

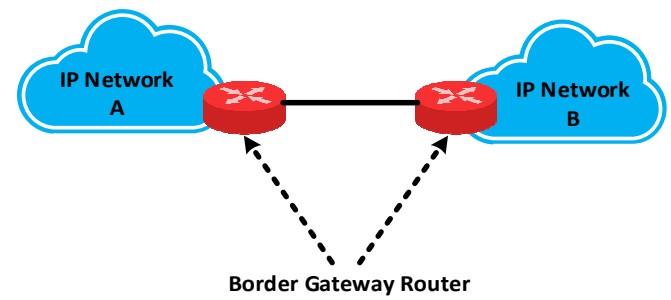

Fig. 5 Private Peering

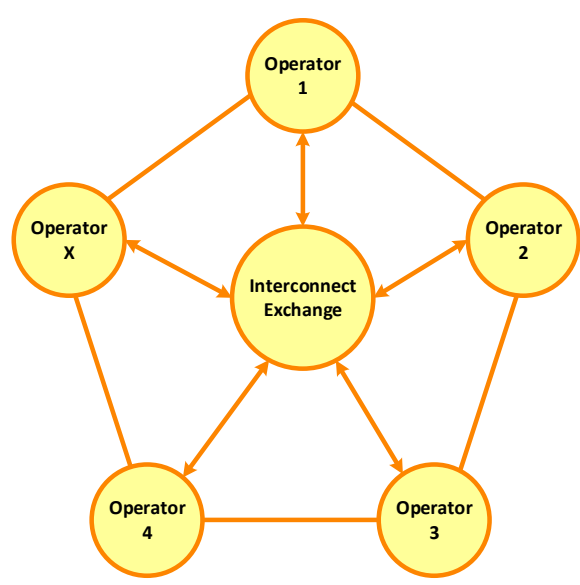

Fig. 6 Public Peering

Internet transit is a system in which one Internet Service Provider (ISP) provides connectivity to all destinations on the global Internet. Transit providers (ISP cores) manage traffic according to the internet routing tables and bring traffic to ISP transit customers who pay the service provider. The characteristics of Internet transit is that the technical and business arrangements are made bilaterally. Most transit agreements state that transit providers will bring traffic to/from other customers and to/from any destinations on the Internet. For example, a transit provider gives the client access to all network routes in the routing table. Transit providers bring traffic to or from the third parties to customers (endpoints). The price is set for access to the entire Internet, usually by volume, measured in Mbps. From a customer perspective, the relationship is simple; paying customers and transit providers give access to the entire Internet, including Service Level Agreement (SLA) supports, installation \& Network Operations Centre (NOC). Fig. 7. shows the transit provider concept that serves several ISPs.

\section{Interconnection on Managed Network}

Interconnection on Managed Network is a term used to separate IP interconnection from other IP-based services such as Over-The-Top (OTT). Services on a managed network do not use any public Internet networks but instead use separate networks; hence the traffic and service quality can be controlled.

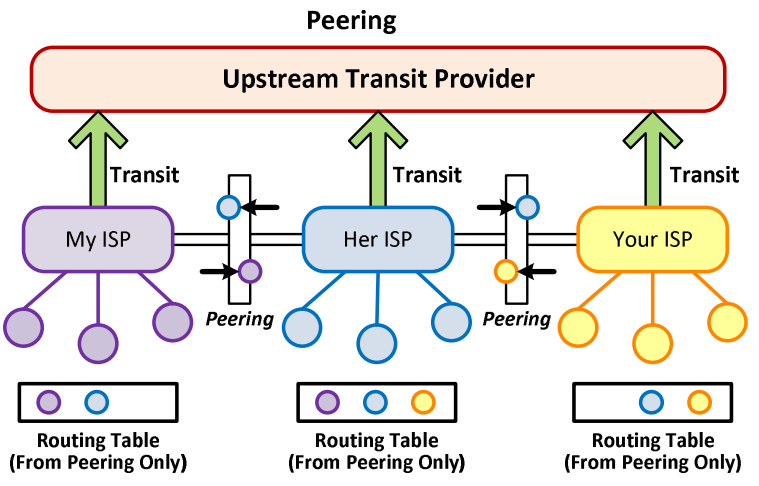

Fig. 7 Transit interconnection [10]

Traffic and information flows are maintained because the network is private. For example, in America, VoIP providers separate voice packages from other services to maintain quality. AT \& T and Verizon do this. Overall, mostly VoIP traffic is of managed services. The interconnection on a managed network consists of physical and logical interconnections of the operator network that occur to originate, terminate, and/or exchange traffic on Managed VoIP services, including the functions and features associated with it. In general, the interconnection of managed IP services can be distinguished into direct interconnection, indirect interconnection, and hubbing (indirect interconnection through multiple providers). Fig. 8. shows some types of managed IP interconnections.

In the direct interconnection model, carriers carrying initial traffic will be charged interconnection fees by the destination operator according to the existing regulatory policies. Under the current interconnection regime, interconnection rates for termination based are regulated by the government on a cost-based approach. Fig. 9. shows the concept of a direct interconnection. 
Indirect interconnection can occur in international connectivity or wide-area connectivity. Fig. 10. shows the concept of an indirect interconnection. Hub is a termination model for IP traffic exchange between customers from different mobile and fixed operators and other providers through IP-to-Network based Interfaces. GSM Association developed the Interconnection Hub. The Interconnection Hub is not meant to replace or compete with the Internet but to offer alternative options for service providers.

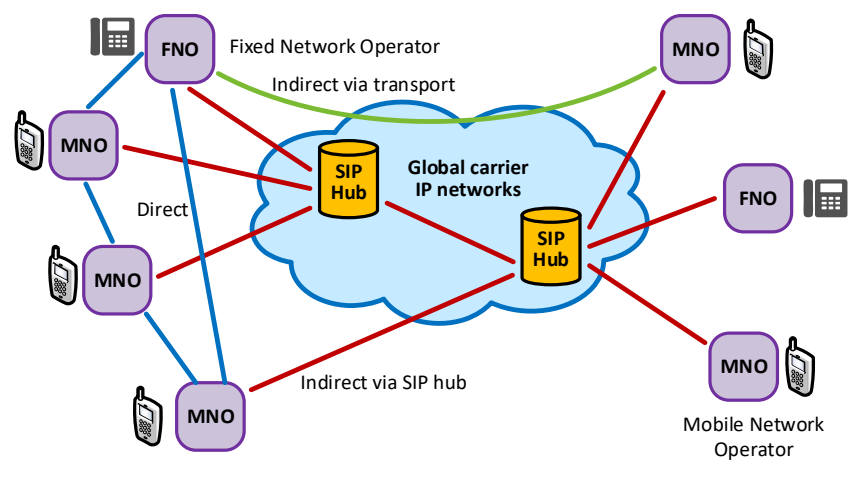

Fig. 8 Types of managed IP interconnections [11]

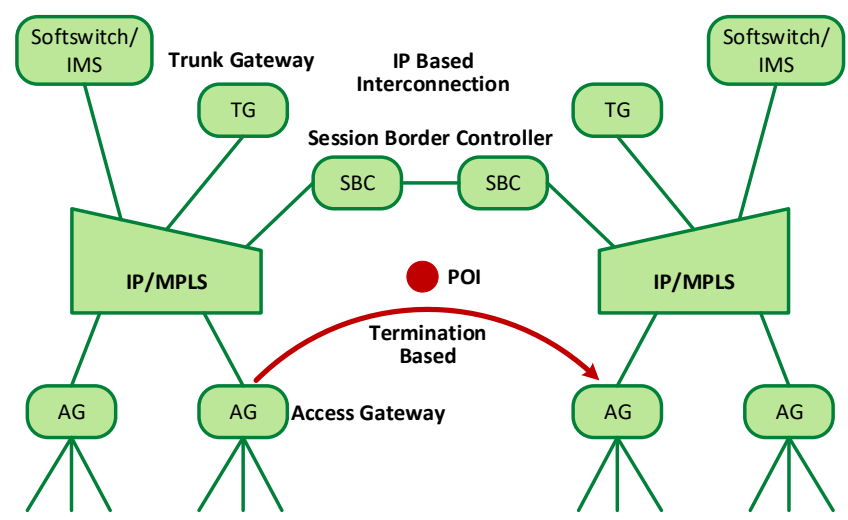

Fig. 9 Direct Interconnection [12]

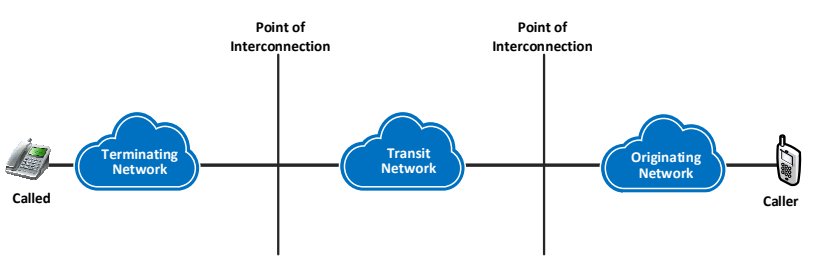

Fig. 10 Indirect interconnection

The purpose of the Interconnection Hub is to provide interoperability of IP-based services between all types of providers within a commercial framework that allows all parties in the value chain to receive commercial profits. Commercial relationships are supported by service level agreements that ensure performance, quality, and security, called Interconnection Bid Documents. Fig. 11. shows the hubbing interconnection IP.

\section{IP-Multimedia Subsystem (IMS) Interconnection}

In the interconnection of managed IP services, the implementation can be done using IMS. It is a telecommunication model that enables standard access based on IP-network to various network services. Created by ETSI TISPAN and later developed by $3 \mathrm{GPP}$, IMS provides an opportunity for fixed and mobile operators to incorporate services into a core network. It has features for interconnecting both mobile and fixed networks, provision of integrated voice and video services, and provision of new legacy SMS services and message services. Four cases are classified and can cover all call scenarios.

Case-1: From IMS to IMS without fixed/mobile interworking, for example, a call originating from a mobile IMS 4G terminated on IMS 4G mobile. Fig. 12 illustrates the case-1 of IMS-IMS interconnection without fixed/mobile internetworking. In this case, the interconnection characteristics are as follows:

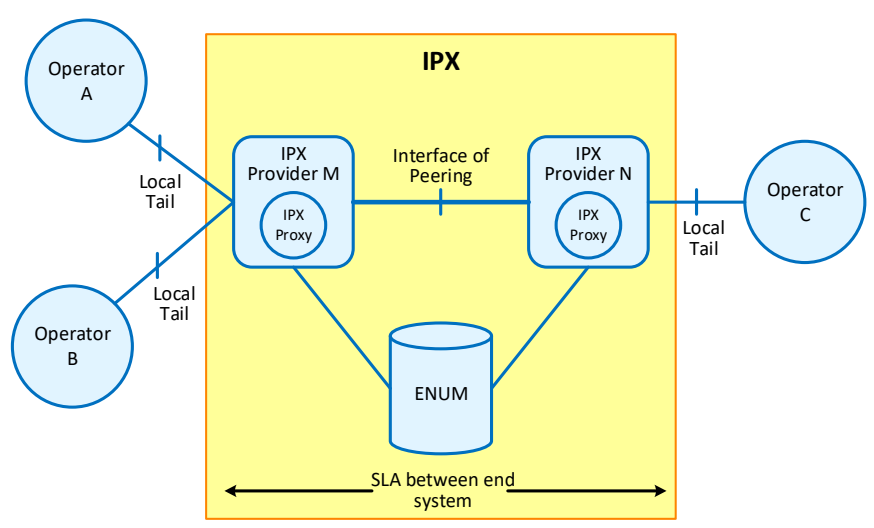

Fig.11 Hubbing IP Interconnection [13]

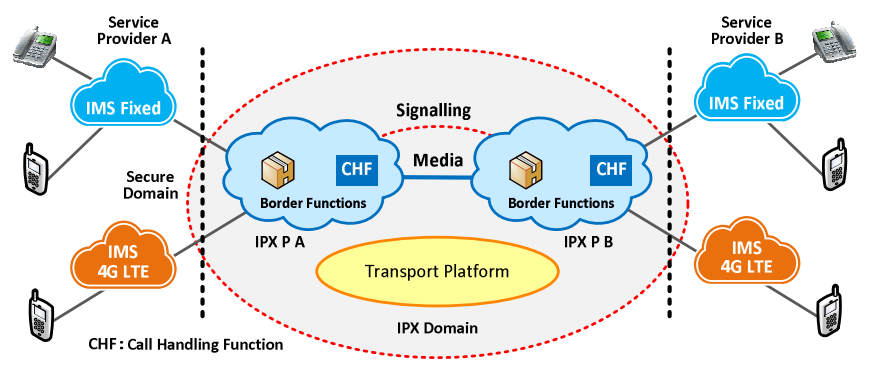

Fig. 12 Interconnection of IMS-IMS without fixed/mobile Internetworking [14]

- Service: voice services can be available in different variants depending on the device (mobile or fixed)

- Signaling: supports STI signaling standards as per 3GPP specification TS 29.165

- Transcoding: for fixed networks, no transcoding is required.

- Addressing: 2 schemes such as Tel-URI or SIP-URI user, and SIP-URI alphanumeric.

Case-2: IMS - IMS with fixed/mobile interworking, e.g. a call coming from a fixed IMS network terminated on a $4 \mathrm{G}$ IMS mobile network and vice versa. Fig. 13. shows an illustration for case 2 . In this case, the interconnection characteristics are as follows: 
- Service: voice services can be available in different variants depending on the device (mobile or fixed)

- Signaling: supports STI signaling standards as per 3GPP specification TS 29.165

- Transcoding: transcoding is required on either side of the carrier or one of the IPX.

- Addressing: 2 schemes such as Tel-URI or SIP-URI, and SIP-URI alphanumeric.

Case 3: from legacy network to IMS or vice versa, for example, a call is coming from a non-IMS network on IMS or mobile IMS 4G and vice versa. Fig. 14 illustrates the interconnection between an existing legacy network and an IMS. In this case, the interconnection characteristics are as follows:

- Service: basic voice services are available

- Signaling: there is interworking signaling from the ISUP to the SIP IMS.

- Transcoding: transcoding is required on either side of the carrier or one of the IPXs.

- Addressing: addressing schemes can be used is E.164.

- Case 4: a call is originating from a fixed on a legacy VoIP network and vice versa. Fig. 15 illustrates case 4. In this case, interconnection characteristics are as follows:

- Service: voice service in different variants. The interoperability of additional services may not necessarily occur.

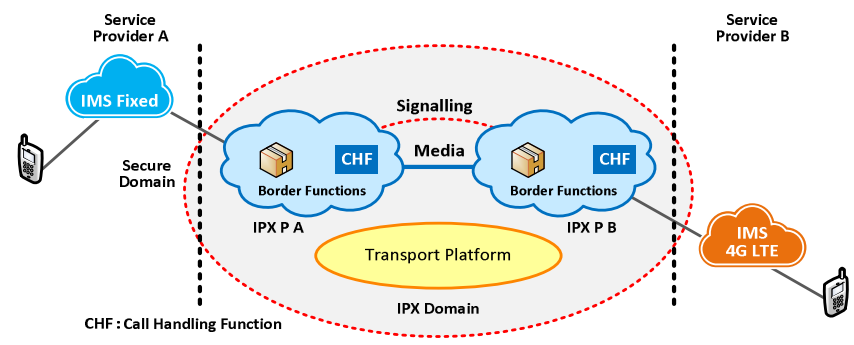

Fig. 13 IMS-IMS interconnection with fixed/mobile internetworking [14]

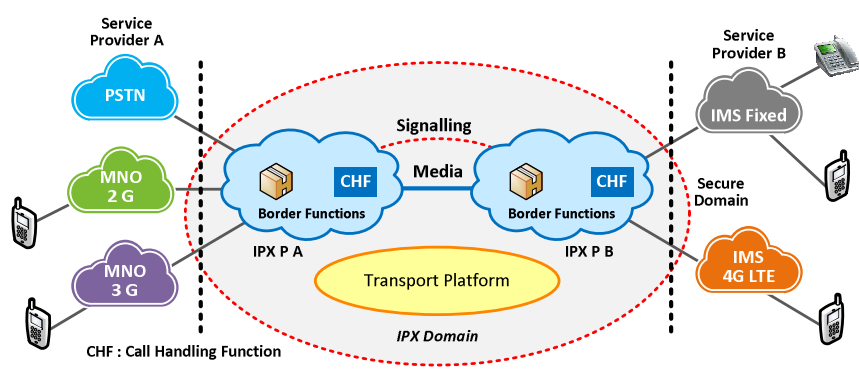

Fig. 14. Interconnection of the legacy network to IMS network [14]

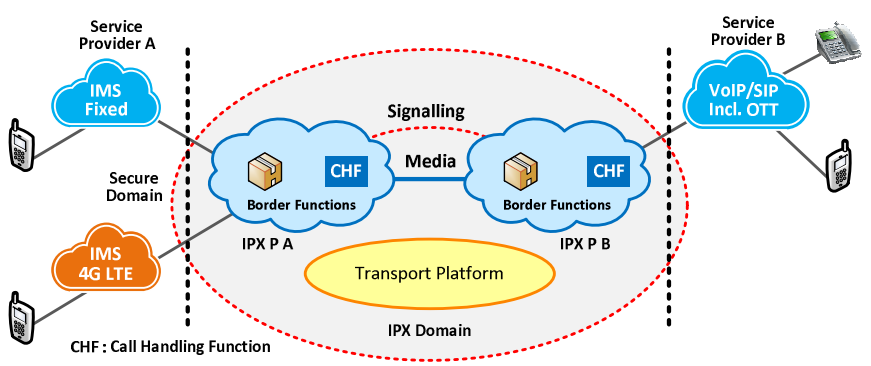

Fig. 15. Call from IMS to legacy network [14]
- Signaling: interworking and interoperability are done at the first IPX

- Transcoding: transcoding is required on either side of the carrier or one of the IPXs.

- Addressing: 2 schemes such as Tel-URI and SIP-URI.

\section{RESULTS AND DISCUSSIONS}

\section{A. Signaling Standard}

In IP Telephony, there are different types of signaling protocols used, such as H.323, SIP, MGCP and H.248. Nevertheless, SIP is one of the most widely recognized standards of signaling. The 3rd Generation Partnership Project (3GPP) has proposed an IMS, which is an addition to the Packet-Switched (PS) network system. IMS supports multimedia services, on the top of IP, as well as interoperability with analog phone services. IMS is a framework that enables richer IP services and applications in addition to packet data delivery.

3 GPP has selected Session Initiation Protocol (SIP) as the signaling standard between end-point and IMS as well as between entities within the IMS itself. SIP performs the process of forming, modifying, and ending multimedia communication sessions. SIP is used for interactive multimedia services such as internet phone, instant messaging, multimedia conferencing, and distributed games on both wireless and wired networks. SIP itself is a textbased application-level protocol with a request-response model. It is designed by the Internet Engineering Task Force (IETF). 3GPP has adopted and modified SIP to complement the SIP core protocol (e.g. new header, as in 3GPP TS 24.229). This addition is done for various reasons, such as for billing system, security and management. In 3GPP, SIP servers and proxies are more complex than are used on the Internet. Nevertheless, all 3GPP extensions are developed in conjunction with IETF, because of collaboration between IETF and 3GPP. As the development of the IETF core-SIP protocol, SIP used in IMS can be operated with SIP protocols based on the IETF specification.

Several types of SIP can be used on the NGN network, such as SIP-IETF (RFC 3261), SIP-IMS (IETF and 3GPP), SIP-T (Telephony) and SIP-I (ISUP). SIP is the protocol that occupies the application layer. This position can be described in the SIP Stack protocol diagram. Some of the protocols contained in the IP Telephony environment are as follows.

- User Datagram Protocol (UDP); it is a protocol contained in the TCP/IP protocol packet used to transmit data units called datagrams over IP networks.

- Transport Layer Security (TLS); it supports other protocols to provide over IP networks, namely: TLS Handshake, Record Protocol, and security.

- SDP (Session Description Protocol); it describes multimedia sessions between user agents.

- MGCP (Media Gateway Control Protocol); it is the protocol that controls media gateway providing access to the PSTN network.

- SAP (Session Announcement Protocol); it is a protocol that performs multicast and TRIP (Telephony Routing over IP) sessions to allocate the best gateway 
between PSTN and the Internet, and it is used as a protocol to allocate multicast addresses.

SIP signaling operations can be simplified into SIP addressing, determining SIP Server location, SIP transaction, SIP invitation, specifying user location, and changing sessions performed. Another type of SIP is SIP-IMS. There are two major components in IMS, i.e., signaling component and bearer component. The former consists of an important function for control call session. SIP is used as a signaling protocol in the IMS platform based on RFC 3261. It has functions to establish, manage, and terminate sessions as well as organizing participants within the IMS architecture. There are currently two standard systems available to connect the SIP soft switch domains and the traditional PSTN, SIP-T protocol packets from the IETF and SIP-I compiled by ITU-T.

SIP-T is an extension of SIP, which inherits SIP features such as flexibility and provides additional supports for phone applications, making it highly suitable for IP networks. SIP$\mathrm{T}$ allows SIP messages to carry ISUP (ISDN User Part) signals. In SIP-T, there are three models for interworking between SIP and ISUP end-to-end, i.e., PSTN-PSTN calls through SIP networks, SIP-PSTN calls, and PSTN-SIP calls. SIP-T is defined in RFC3372 from the IETFMMUSIC working group. The SIP-T protocol package contains RFC3372, RFC2976, RFC3204 and RFC3398. SIP-T provides encapsulation (defined by RFC3204) and mapping (defined by RFC3398) for interworking between SIP and ISUP.

SIP-I is an extension of SIP, which is designed by ITU in collaboration with IETF that allows IP networks to provide services supported by the ISUP network. RFC Q.1912.5 defines the interworking between Bearer Independent Call Control Protocol (BICC)/ISUP and 3GPP SIP, between BICC /ISUP and SIP, and between BICC/ISUP and SIP-I. RFC TRQ.2815 defines the technical requirements for interworking between BICC/ISUP and SIP. SIP-I uses standard functions defined by the IETF, which includes not only basic call interworking but also interworking of BICC/ISUP additional services.

SIP can connect users using IP networks (LAN and WAN, public Internet backbone networks, 2.5G, 3G and Wi-Fi cellular networks) and IP equipment (phones, PCs, Personal Digital Assistants (PDAs) and other mobile devices) thereby improving the communication mode between the company and the user. The advantage of SIP lies in its ability to incorporate these functions into integrated communications services.

SIP-I and SIP-T are both the development of SIP. SIP-I uses many IETF standards and is richer in content than SIPT. SIP-I not only contains interworking for basic calls but also interworking of other services. In addition to the interworking of call signaling, SIP-I also pays attention to other things such as resource reservation, media information conversion, and interworking between fixed-line SIP/3GPPSIP and BICC/ISUP. Most importantly, the SIP-I inherits SIP's advantages such as clarity, precision and elaboration derived from the traditional ITU-T recommendations, and it is better than SIP-T in operation. SIP-I has been regarded as an interworking core protocol between the soft switch and traditional telecommunication network by 3 GPP.

\section{B. Coding Standard}

The human voice is transformed into a digital signal and transmitted over the network Using a coder/decoder (codec), a device for converting digital data formats from original data into data of smaller size without significantly reducing voice quality. The traditional signaling protocol for voice (ISUP) uses the G.711 codec as the standard defined by ITU-T. This type of codec is commonly used in telephony networks. In its development, there are several types of codecs used, such as G.711 A-law, G.722, G.729, G.729a (ITU-T Standard) and DTMF, EFR, and AMR (3GPP Standard).

G.711 is an audio compression ITU-T standard used on a telephone network. It was released in 1972 and used in many specifications, such as H.320 as well as H.323. It can also be used for fax communications over IP networks. G.711, often called Pulse Code Modulation (PCM), is an audio codec for a narrowband network that provides good audio quality at 64 $\mathrm{kbit} / \mathrm{s}$. It works in the $300-3,400 \mathrm{~Hz}$ frequency range. G.711 has very low overheads due to a simple algorithm for converting voice signals into digital formats, but the result is in poor network efficiency due to inefficient bandwidth usage. The G.711 standard has been continuously improved by adding new functions such as G.711.0 and G.711.1 for lossless data compression and improving audio quality by increasing bandwidth. G.711 has two versions, namely A and $\mu$-law. $\mu$-law and A-law have differences in the dynamic range of output. When the voice is very soft that A-Law will sound better than $\mu$-law because $\mu$-law has a greater dynamic range compare to A-Law.

ITU-T recommends G.729 codec for speech signals encoding at $8 \mathrm{kbps}$ data rates using Exclusive Linear Prediction (CS-ACELP) Algebra Code Conjugation Code. It uses 8000 samples per second while using a 16-bit linear PCM as an encoding method. In the G.729 codec, the data payload is compressed to $8 \mathrm{kbit} / \mathrm{s}$, which gives 8 times greater capacity for the same connection as G.711. The G.729 codec is suitable for limited bandwidth, such as ADSL VoIP and satellite connections. The use of the G.729 VoIP application is easier by occupying a lower bandwidth of 8kbps. G.729.1 allows measurable data rates of ranging between 8 and $32 \mathrm{kbps}$. And G.729.1 is a high speed and wide bandwidth encoding algorithm, which can be operated with G.729A, G.729B and G.729 codecs.

The other codecs are Opus and Adaptive Multi-Rate (AMR). Opus is a codec designed according to IETF RFC 6716 standard. Opus can handle various audio applications, including VoIP and videoconferencing. This codec can compress the voice data until it reaches the size when it can be sent at bitrate $6 \mathrm{kbit} / \mathrm{s}$. AMR is used for speech coding that its format is designed based on ISO by 3GPP. In addition to the standard codecs defined by ITU-T and 3GPP, there are other codecs already implemented for voice coding. Here are some commonly used codecs: ACELP, AC-3, ALAC and iLBC.

In various surveys and studies, it is shown that Codec G.711 and G.729 are most widely used because it provides better call quality and better bandwidth usage. The G.711 
codec is widely used in high bandwidth network environments, while G.729, with higher compression rates, is suitable for use in limited bandwidth environments. Based on specifications, G.729 provides low data rates. Unfortunately, it has Intellectual Property (IP) rights that need to be licensed. Meanwhile, G.711 does not need any license, so most devices use G.711, and it has simple interoperability.

\section{Quality of Service $(Q o S)$}

For guaranteeing the QoS of VoIP, subscribers must receive voice transmission better than basic phone service meaning high-quality voice transmission must be maintained consistently. Similar to other real-time applications, VoIP has a sensitivity to bandwidth and delay. In order that the VoIP transmission is received correctly by the receiver, the voice packet should not be discarded hence excessive delay or jitter (delay variation) will not be too high.

VoIP can guarantee high-quality transmission while its packets are given priority in the network rather than other packets. VoIP packet traffics has to be guaranteed In order to be used with acceptable sound quality levels. It needs certain threshold values in some parameters such as latency, bandwidth, and jitter. QoS will provide better services by supporting the following features: Support priority bandwidth for VoIP, increase packet loss characteristics, Avoid network congestion, Arrange traffic on the network, and it has dedicated bandwidth.

IP-based interconnects require QoS guarantees to compensate for lack of IP layer connections that have the best effort communication mode without any connection setup at the beginning. Packages are sent from a source to a destination directly without any connection setup. On that layer, there is no mechanism to guarantee service quality. This needs to be supported by the above layers by conducting a mechanism that can guarantee the QoS required.

Some QoS parameters used to ensure the availability of IP-based Interconnection services is:

- One-way Delay (end-to-end) (G.114): the time required by a single packet from the source to get to the destination. The default value is $<150 \mathrm{~ms}$.

- $M O S$ (LQO) (end-to-end): The Mean Opinion Score (MOS) is a measurement of customer satisfaction on the quality of communication services with values ranging from 1 and 5 ( 1 = Quality of the head, $5=$ Very good quality). Default value> 4.0. Listener Quality Only (LQO) is a measurement of the quality perceived by the telephone receiver. Default value> 4.0.

- $R$-factor: the transmission rating factor $\mathrm{R}$ (R-factor) measures service quality like the measured MOS with the tool. The default value> $70 \%$.

- The codec used. The standard codecs are G.107, G.168, G.711 and G.729.

- Call set-up time: the time required to build communication. The default value is $<3 \mathrm{~s}$.

- NER: The Network Effectiveness Ratio (NER) is the network's ability to send calls from the caller to the called phone. The default value is $>99.3 \%$.
- The Answer Seizure Ratio (ASR). It is the called phone responds compared to the total number of calls. The default value is $>50 \%$

- Availability of the interconnection: availability of network interconnection for the end-to-end communication process. The default value is $>99.5 \%$

- Packet Loss Ratio (PLR): it is the number of packets lost compared to the total packet sent. The lower, the better.

- Packet transfer delay: it is time it takes a packet from the source to reach the destination. The lower, the better.

\section{Numbering and Addressing}

Numbering is one component that plays an important role in the telecommunications industry, where numbers are used as customers' identities and customers' addresses to recognize and transmit traffic and services. The numbering system in the telecommunication network in Indonesia currently refers to ITU-T Recommendation E.164. Telecommunication systems on fixed networks, telecommunication systems in mobile networks, as well as national services, use the same structure.

Based on the ITU-T recommendation, the international number for the subscriber consists of the State Code and National Number (Significant) which includes the National Destination Code [NDC]), including two numbering categories, as follows:

- Numbering that contains geographical indication: in this case, NDC serves as a Regional Code that characterizes a numbering area.

- Numbering that does not contain any geographical indication: in this case, the NDC serves as a Network Access Code that characterizes the type of network, or as a Service Access Code that characterizes the type of service.

The development of network technology is currently designed by world bodies, such as ITU-T, IETF and 3GPP, leading to an NGN concept. NGN will have the capability to provide services and of using a variety of broadband transport technologies and independent service functions of transport technology. Improved aspects of service at NGN are in terms of coverage and quality. NGN service coverage is much broader than the existing telecommunications network, including broadband and narrowband services, real-time, non-real time, one-to-one, multicasting, broadcasting, and multimedia. Other services include human-to-human, human-to-object, and object-to-object communications. With the transformation of the entire network system shifted to the new network, the numbering system needs to be adjusted to meet the new network conditions.

In IP-based networks, some addressing characteristics that must be met are as follows:

- Support both dynamic and fixed addressing modes.

- Addressing systems, naming systems and directory services can be implemented using a one-to-one mapping scheme for each service, or through common mapping schemes covering different services.

- Supports dynamic updates from the naming service database. For example, in the case of a mobile 
terminal, addresses on one or more layers may change dynamically depending on the terminal location, and the database should be able to anticipate this.

By taking various aspects and interconnection requirements into account, the addressing and numbering schemes must meet the following requirements:

- Supports IP addressing schemes.

- Supports IP-based multimedia communication protocol (in the originating and terminating cases) using at least TEL URI (E.164), e.g., tell: +4412345678 and/or SIP URI, e.g., sip: my.name@company.org.

- Addressing scheme also supports various service types, such as unicast, multicast and broadcast.

- Supports a group addressing scheme, which allows multicasting like a conference service.

- Supports other addressing and naming schemes such as private numbering.

\section{E. Security}

Communication between two different systems in an Internet network requires its own security. There are many points that need to be secured from the starting point when the data is sent until the point when the data is accepted and communication established. Some of the mechanisms that can be done to deal with network security issues are as follows:

1) Use of Session Border Controller: a control device used to control signaling and transmission of media data during connection setup, communication, and closure of telephone connections or other communication devices.

2) IP addresses not announced on the Internet: IP addresses are exchanged between operators and their partners on a limited basis.

3) Authentication of BGP and EBGP: a TCP segment data authentication process between peer network. One method for authentication is to use MD5 authentication. Each of the data segments sent from one source to the destination has to be verified first.

4) Security in each internal operator, such as the process of blocking ports and specializing for the right.

5) Installing a Firewall on the border gateway.

6) Building redundant connections for point-to-point, both physically and logically. If one connection gets attacked from outside, the system can use the other connection.

7) Security layer 3 and layer 4:

- Detect and drop non-standard TCP/IP packets.

- Detect and drop non-standard SIP/H.323 messages.

- Traffic settings

- Topology hiding for SIP/H.323 sessions

- Topology hiding for media.

- Session restrictions

- Authentication, Integrity and Confidentiality (SSH)

8) IP address allocation for each operator: the IP address of the operator is determined hence an easy detection of any use of IP address outside the specified address.

9) Control of signaling and media packet access: both signaling and media packages are checked first to ensure no attacks in the packet.

10)Prevent SIP and IP messages from unauthorized senders.

11) Session restriction: session connection establishment is completely selective and thus unauthorized users are not free to establish connections.

\section{2) Prevention of DOS attacks.}

13)Traffic filter: only approved traffic can pass the network. This section has presented key parameters for NGN implementation in Indonesia. Technical specifications for each of the key parameters have been described based on prior experience in other countries. By comparing the success of other countries that have implemented in advance, the risk of implementing NGN in Indonesia will be well managed.

\section{CONCLUSIONS}

The implementation of IP-based NGN network in Indonesia to replace existing various telecommunication interconnection system is a complex process. Planning and preliminary studies are required to ensure effective NGN network implementation process. Based on any experience of implementing NGN infrastructure in many countries, this paper has proposed a technical specification model for the case of NGN implementation in Indonesia. In this proposal, the technical specification models that have been defined are signaling protocols, coding standards, QoS, numbering and addressing, and security. These criteria are key parameters for the effectiveness of NGN implementation.

\section{ACKNOWLEDGEMENT}

Telkom University and Ministry of Communication and Information of Republic Indonesia fund this research. Authors thank all colleagues in Telkom University and the Ministry of Communication and Information.

\section{REFERENCES}

[1] M. Abdurohman, B. S. Nugroho, and A. G. Putrada, "Telecommunication Numbering System Roadmap Towards NGN Era in Indonesia," Indones. J. Electr. Eng. Comput. Sci., vol. 5, no. 2, pp. 363-375, 2017.

[2] BEREC, "Case Studies on IP-based Interconnection for Voice Services in the European Union," Body of European for Electronic Communications (BEREC), Nov. 2015.

[3] K. Knightson, N. Morita, and T. Towle, "NGN architecture: generic principles, functional architecture, and implementation," IEEE Commun. Mag., vol. 43, no. 10, pp. 49-56, Oct. 2005.

[4] Choi, M.J., Hong, J.W.K., "Towards Management of Next Generation Networks.” IEICE. 2007.

[5] Chae-Sub Lee and D. Knight, "Realization of the next-generation network," IEEE Commun. Mag., vol. 43, no. 10, pp. 34-41, Oct. 2005.

[6] M. Callejo-rodriguez and J. Enriquez-Gabeiras, "Bridging the standardization Gap to provide QoS in current NGN

[7] C. Czarnecki, M. Heuser, and M. Spiliopoulou, "How does the implementation of a next generation network influence a telecommunication company," in Proceedings of the European and 
Mediterranean Conference on Information Systems (EMCIS), 2009, pp. 13-14.

[8] GSMA,'Connecting the mobile industry - connecting people”. GSM Association. 2018.

[9] Comptel, "IP-IP interconnection." The competitive communication association.

[10] Zuckerman, E., McLaughlin,A.,'Introduction to Internet Architecture and Institutions". Harvard University. 2003.
[11] Baldwin, J., Ewert, J., Yamen, S."Evolution of the voice interconnect". Ericsson Review. 2010.

[12] Bhawan, M.D., Marg, J.L.N., "Consultation Paper on Migration to IP based Network." Telecom Regulatory Authority of India. 2014.

[13] GSMA, "Inter-Service Provider IP Backbone Guidelines". Official Document IR.34. London. 2011.

[14] i3forum," IMS-Based Services: Technical and Commercial Analysis of International Interconnection and Roaming Services”. IMS Based Service. 2016. 